\title{
The first interferometric detections of fast radio bursts
}

\author{
M. Caleb, ${ }^{1,2,3 \star}$ C. Flynn, ${ }^{2,3}$ M. Bailes, ${ }^{2,3}$ E. D. Barr, ${ }^{2,3,4}$ T. Bateman, ${ }^{5}$ S. Bhandari, ${ }^{2,3}$ \\ D. Campbell-Wilson, ${ }^{3,5}$ W. Farah, ${ }^{2}$ A. J. Green, ${ }^{3,5}$ R. W. Hunstead, ${ }^{5}$ A. Jameson, ${ }^{2,3}$ \\ F. Jankowski, ${ }^{2,3}$ E. F. Keane, ${ }^{6}$ A. Parthasarathy, ${ }^{2,3}$ V. Ravi, ${ }^{2,3,7}$ P. A. Rosado, ${ }^{2,8}$ \\ W. van Straten ${ }^{2,9}$ and V. Venkatraman Krishnan ${ }^{2,3}$ \\ ${ }^{1}$ Research School of Astronomy and Astrophysics, Australian National University, ACT 2611, Australia \\ ${ }^{2}$ Centre for Astrophysics and Supercomputing, Swinburne University of Technology, PO Box 218, Hawthorn, VIC 3122, Australia \\ ${ }^{3}$ ARC Centre of Excellence for All-Sky Astrophysics (CAASTRO), School of Physics, The University of Sydney, NSW 2006, Australia \\ ${ }^{4}$ Max-Planck-Institut für Radioastronomie, Auf dem Hügel 69, D-53121 Bonn, Germany \\ ${ }^{5}$ Sydney Institute for Astronomy (SIfA), School of Physics, The University of Sydney, NSW 2006, Australia \\ ${ }^{6}$ SKA Organisation, Jodrell Bank Observatory, Cheshire, SK11 9DL, UK \\ ${ }^{7}$ Cahill Center for Astronomy and Astrophysics, MC249-17, California Institute of Technology, Pasadena, CA 91125, USA \\ ${ }^{8}$ Monash Centre for Astrophysics, School of Physics and Astronomy, Monash University, VIC 3800, Australia \\ ${ }^{9}$ Institute for Radio Astronomy and Space Research, Auckland University of Technology, Private Bag 92006, Auckland 1142, New Zealand
}

Accepted 2017 March 13. Received 2017 March 13; in original form 2016 November 15

\begin{abstract}
We present the first interferometric detections of fast radio bursts (FRBs), an enigmatic new class of astrophysical transient. In a 180-d survey of the Southern sky, we discovered three FRBs at $843 \mathrm{MHz}$ with the UTMOST array, as a part of commissioning science during a major ongoing upgrade. The wide field of view of UTMOST $\left(\approx 9 \mathrm{deg}^{2}\right)$ is well suited to FRB searches. The primary beam is covered by 352 partially overlapping fan-beams, each of which is searched for FRBs in real time with pulse widths in the range $0.655-42 \mathrm{~ms}$, and dispersion measures $\leq 2000 \mathrm{pc} \mathrm{cm}^{-3}$. Detections of FRBs with the UTMOST array place a lower limit on their distances of $\approx 10^{4} \mathrm{~km}$ (limit of the telescope near-field) supporting the case for an astronomical origin. Repeating FRBs at UTMOST or an FRB detected simultaneously with the Parkes radio telescope and UTMOST would allow a few arcsec localization, thereby providing an excellent means of identifying FRB host galaxies, if present. Up to $100 \mathrm{~h}$ of followup for each FRB has been carried out with the UTMOST, with no repeating bursts seen. From the detected position, we present $3 \sigma$ error ellipses of $15 \operatorname{arcsec} \times 8.4$ on the sky for the point of origin for the FRBs. We estimate an all-sky FRB rate at $843 \mathrm{MHz}$ above a fluence $\mathcal{F}_{\text {lim }}$ of $11 \mathrm{Jy}$ ms of $\sim 78$ events sky ${ }^{-1} \mathrm{~d}^{-1}$ at the 95 per cent confidence level. The measured rate of FRBs at $843 \mathrm{MHz}$ is two times higher than we had expected, scaling from the FRB rate at the Parkes radio telescope, assuming that FRBs have a flat spectral index and a uniform distribution in Euclidean space. We examine how this can be explained by FRBs having a steeper spectral index and/or a flatter $\log N-\log \mathcal{F}$ distribution than expected for a Euclidean Universe.
\end{abstract}

Key words: instrumentation: interferometers-methods: data analysis-surveysintergalactic medium - radio continuum.

\section{INTRODUCTION}

Fast radio bursts (FRBs) are a relatively new class of radio transient that are short, bright and highly dispersed. The pulses are typically of durations of a few milliseconds, and exhibit a dispersion sweep characteristic of propagation through a cold diffuse plasma

^E-mail: manishacaleb@gmail.com
(Lorimer et al. 2007; Thornton et al. 2013). The dispersion measures (DMs) of these pulses are significantly higher than the contribution from the line of sight through the Galactic interstellar medium (ISM), suggestive of a cosmological origin in which the large DMs are due to passage through the intergalactic medium (IGM). If they are at cosmological distances, their inferred intrinsic energies $\left(>10^{31} \mathrm{~J}\right)$ and brightness temperatures $\left(T_{\mathrm{b}}>10^{33} \mathrm{~K}\right)$ necessitate a coherent emission mechanism, while the short durations of the pulses suggest a very compact source of origin (Dennison 2014; 
Luan \& Goldreich 2014). The 18 FRBs published to date [refer to the FRBCAT repository ${ }^{3}$ for the complete list (Petroff et al. 2016)] have been discovered in either post-processing of archival surveys or, in real time, using the Parkes radio telescope with the exception of two, detected at the Arecibo (Spitler et al. 2014) and Green Bank telescopes (GBT) (Masui et al. 2015). All but one of the bursts have been found at $1.4 \mathrm{GHz}$, with the exception being the GBT burst, which was seen at $800 \mathrm{MHz}$.

The observed FRB all-sky rate is very high. Champion et al. (2016) derive a rate of $7_{-3}^{+5} \times 10^{3}$ events $\mathrm{sky}^{-1} \mathrm{~d}^{-1}$ at $1.4 \mathrm{GHz}$ for bursts between 0.13 and $1.5 \mathrm{Jy} \mathrm{ms}$ in fluence and widths in the range $0.128-16 \mathrm{~ms}$. The high FRB rate is a major constraint on theories for their origin. Until recently, such theories have generally assumed they are cataclysmic events, in which the progenitor is obliterated. However, one FRB is now known to repeat in a non-periodic manner (FRB 121102; Spitler et al. 2016), opening up possibilities for other progenitor models. Following the discoveries reported in this paper, Chatterjee et al. (2017) have achieved sub-arsecond localization of the FRB 121102 using radio interferometric observations from the Very Large Array. The source has been localized to a $m_{r^{\prime}}=25.1 \mathrm{AB}$ mag low-metallicity, star-forming dwarf galaxy at $z=0.19273(8)$ (Tendulkar et al. 2017). The precise localization shows that the source is either co-located with a $180 \mu \mathrm{Jy}$ active galactic nucleus or an unresolved type of extragalactic source. However, the exact nature of the FRB progenitor is still unknown.

Despite concerted follow-up efforts for almost all FRBs, this remains the only FRB seen to repeat. These efforts have been quite substantial. For instance, $\approx 80 \mathrm{~h}$ of follow up for the Lorimer burst (Lorimer et al. 2007), $\approx 80 \mathrm{~h}$ for FRB 131104 (Ravi, Shannon \& Jameson 2015) and $\approx 110 \mathrm{~h}$ of selected FRB positions (Petroff et al. 2015) at the Parkes radio telescope yielded no repeats. This suggests the possibility of there being two independent classes of FRBs - repeating and non-repeating - with two classes of possible progenitors (Keane et al. 2016). Progenitor theories include flaring magnetars (Lyubarsky 2014), giant pulses from pulsars (Cordes \& Wasserman 2016; Connor, Sievers \& Pen 2016a; Lyutikov, Burzawa \& Popov 2016), binary white dwarf mergers (Kashiyama, Ioka \& Mesaros 2013), neutron star mergers (Totani 2013) and collapsing supramassive neutron stars (Falcke \& Rezzolla 2014). It is possible that the lack of repetition of pulses for the FRB discoveries at the Parkes radio telescope is merely due to limited sensitivity and follow-up time, and that all FRBs have a common origin (Scholz et al. 2016). FRB 010724 is an exception to this; however, its extreme brightness $(\sim 30 \mathrm{Jy})$ far outweighs the lower gain of Parkes relative to Arecibo, so that one cannot infer its lack of repeat bursts is due to limited sensitivity. Recently, Ravi et al. (2016) have reported the detection of FRB 150708, which is of comparable brightness ( $\sim 12 \mathrm{Jy}$ ) to FRB 010724 , and exhibits 100 per cent polarization and suggests weak turbulence in the ionized IGM. DeLaunay et al. (2016) have associated a $\gamma$-ray transient with the FRB 131104 discovered by Ravi et al. (2015). However, Shannon \& Ravi (2017) in contrast, report on the discovery of a variable source (consistent with an AGN) temporally and spatially coincident with the FRB 131104 but not spatially coincident with the $\gamma$-ray burst, and rule out the association of the $\gamma$-ray burst with the FRB using probabilistic reasoning.

Most published FRBs have been detected with single dish antennas, with relatively poor angular resolution, and we are unable to indisputably rule out a near-field or atmospheric origin for the one-off events until now. The FRB detections made with the multibeam receiver at the Parkes radio telescope however, are likely to originate at $\gtrsim 20 \mathrm{~km}$ (Vedantham et al. 2016). Also FRB 150418 has been proposed to be associated with a galaxy at $z \sim 0.5$. However, this association has been called into question by Williams \& Berger (2016) and Vedantham et al. (2016), and other models like giant pulses from extragalactic pulsars which could account for the excess DM in the local environment, have been proposed (Connor et al. 2016a). Better localization during discovery in the radio requires an interferometric detection.

In a companion paper, we describe how the Molonglo Observatory Synthesis Telescope (sited near Canberra in Australia) is currently undergoing a major upgrade, with the addition of a stateof-the-art correlator to transform it into an FRB finding machine the UTMOST (Bailes et al., submitted). Two FRB searches were performed with UTMOST in 2015 during the upgrade, when the system was operating at a small fraction of the final expected sensitivity, and only yielded an upper limit of the FRB rate (Caleb et al. 2016b).

We have now undertaken a third FRB survey at UTMOST and discovered three FRBs. These are the first FRBs observed with an interferometer, further strengthening the case for an astronomical origin in addition to the detections at other telescopes and in the expected number of beams at Parkes for far-field events, as detection with UTMOST implies the events are in the far-field region $\gtrsim 10^{4} \mathrm{~km}$. Section 2 of this paper briefly outlines the telescope specifications, survey properties and the transient detection pipeline. We present the bursts' properties and their follow-up observations and localization areas in Section 3. The event rate estimates of the FRBs at $843 \mathrm{MHz}$ based on the detections of the three FRBs and constraints on their spectral index are detailed in Section 4 followed by our conclusions in Section 5 .

\section{UTMOST SPECIFICATIONS AND SURVEY PROPERTIES}

The UTMOST consists of an east-west (E-W) aligned cylindrical paraboloid divided into two 'arms' (separated by a 15-m gap), each 11.6-m wide and 778-m long, with 7744 right circularly polarized ring antennas operating at $843 \mathrm{MHz}$ on a line feed system at its focus. Groups of 22 consecutive ring antennas (these groups are termed 'modules') are phased to the physical centre of the module, forming 352 unique inputs (each with a beam $4.0 \times 2.8 \mathrm{FWHP}$ ) that are then beamformed (Bailes at al., submitted). We operate the telescope by tilting the arms north-south and steering the ring antennas east-west by differential rotation. UTMOST can access the sky south of $\delta=+18^{\circ}$ with the east-west steering limited to $\pm 60^{\circ}$. The telescope's field of view, sensitivity and high duty cycle make it a near ideal survey instrument for finding FRBs and other radio transients. Since late 2015 , we have been using UTMOST to search for fast radio transients for an average of $18 \mathrm{~h}$ a day, while simultaneously timing more than 300 pulsars weekly (Bailes et al., in preparation, Jankowski et al., in preparation).

In FRB search mode, the 4.0 FWHP of the primary beam is tiled in the E-W direction by 352 elliptical, coherent, tied-array beams (called 'fan-beams' or FBs, each 46 arcsec wide), spaced 41 arcsec apart and overlapping at very close to their half power points at $843 \mathrm{MHz}$. In the N-S direction, the resolution of the FBs is the same as that of the primary beam $(\approx 2.8)$. The FBs are numbered from 1 to 352 running from east to west across the primary beam, with FB 177 directly centred on boresight. The sensitivity of the telescope to bursts can be estimated using the radiometer equation:

$S_{\min }=\beta \frac{\left(\mathrm{S} / \mathrm{N}_{\min }\right) T_{\mathrm{sys}}}{G \sqrt{\Delta v W N_{\mathrm{p}}}}$ 
where $S_{\min }$ is the minimum detectable flux for a threshold signalto-noise $\mathrm{S} / \mathrm{N}_{\min }, \beta$ is the digitization factor, $\Delta v$ is the bandwidth in $\mathrm{Hz}, N_{\mathrm{p}}$ is the number of polarizations ( $N_{\mathrm{p}}=1$ for UTMOST as it is right circularly polarized only), $W$ is the pulse width in $\mathrm{ms}$, $T_{\text {sys }}$ is the system temperatures in $\mathrm{K}$ and $G$ is the system gain in $\mathrm{K} \mathrm{Jy}^{-1}$. We define $\mathrm{S} / \mathrm{N}$ as the ratio of the sum of the on-pulse flux to the product of the rms of the off-pulse flux and square root of number of on-pulse bins $\left(\mathrm{S} / \mathrm{N}=\frac{I_{\text {on }}}{\sqrt{\mathrm{nbin}} I_{\mathrm{off}}}\right)$. For the fully upgraded instrument, we expect $S_{\min }=1.6 \mathrm{Jy} \mathrm{ms}$ for a $10 \sigma 1$-ms wide pulse, $3.5 \mathrm{~K} \mathrm{Jy}^{-1}$ gain, $100 \mathrm{~K}$ system temperature and $31.25 \mathrm{MHz}$ bandwidth. The system bandwidth is however only about half of the initially anticipated $31.25 \mathrm{MHz}$ bandwidth, as the ring antennas have a significant roll-off in sensitivity away from $843 \mathrm{MHz}$. This has been measured using integrated pulses from the pulsar J1644-4559. We find that on average $\sim 86$ per cent of the total $\mathrm{S} / \mathrm{N}$ is concentrated in the upper half of the band $(\sim 836-850)$ as the antennas are tuned to maximum sensitivity at $843 \mathrm{MHz}$. We adopt a bandwidth of $16 \mathrm{MHz}$ for the sensitivity calculations in the paper, to be conservative.

During the upgrade, we characterize the system sensitivity by a fraction of the final expected gain $\epsilon$. This factor encompasses systemic losses due to (1) pointing errors (from physical misalignment in the modules $\mathrm{N}-\mathrm{S}$, and phasing errors in the antenna system $\mathrm{E}-$ $\mathrm{W})$, (2) self-generated radio frequency interference (RFI) mainly due to improperly shielded electronics in the receiver boxes near the telescope, (3) coherent noise in the receiver boxes, which affects some sets of adjacent modules, and other inefficiencies in the system performance that we are still characterizing, such as systematic errors in the phase/delay solutions across the interferometer (Bailes et al., in preparation).

At present (2016 October), we estimate $\epsilon \approx 0.14$, based on observations of strong calibrators of known flux densities and a number of high DM pulsars with relatively stable flux densities. This implies an effective $T_{\text {sys }}$ of $400 \pm 100 \mathrm{~K}$. This is significantly higher than the system temperature seen on the best performing modules, which can be as low as $100 \mathrm{~K}$. We note that $\epsilon$ can vary from day to day as modules are either serviced in the field or have electronics maintenance in the workshops, and typically lie in the range $0.15<\epsilon<0.20$. Occasionally, if only one arm is operational, we have the option to continue surveys at half sensitivity (i.e. $0.07<\epsilon<0.10$ ). The telescope can access the southern sky for $\delta<+18^{\circ}$, and for most parts of the sky we tend to observe reasonably close to the meridian, in order to maximize sensitivity. The sensitivity is reduced by projection effects away from the meridian.

In 2015 November, we commenced our third FRB survey 'V3.0'. It ran for a total of $159.0 \mathrm{~d}$ on sky (between 2015-11-01 and 201611-30), at $\epsilon \approx 0.14$ of the final target telescope sensitivity. Our fluence limit of the survey, that is the fluence of the narrowest detectable pulse $\mathcal{F}_{\text {lim }}$ can be parametrized as

$\mathcal{F}_{\lim } \approx 11\left(\frac{W}{\mathrm{~ms}}\right)^{1 / 2} \mathrm{Jy} \mathrm{ms}$

where, $11 \mathrm{Jy}$ is the UTMOST flux limit for $\mathrm{S} / \mathrm{N}=10, G=3.0 \mathrm{~K}$ $\mathrm{Jy}^{-1}, \Delta v=16 \mathrm{MHz}, W=1 \mathrm{~ms}, N_{\mathrm{p}}=1$ and $T_{\text {sys }}=400 \mathrm{~K}$. It should be noted that this is not the same as the fluence completeness limit $\mathcal{F}_{\text {complete }}$. Between $\mathcal{F}_{\text {lim }}$ and $\mathcal{F}_{\text {complete }}$, we are incomplete and not all FRBs with fluences in this range are detectable. This incompleteness region corresponds to the pink shaded region in Fig. 7. The two previous surveys (V1.0 and V2.0) reported in Caleb et al. (2016b) yielded no FRB events. Relative to V3.0, V1.0 ran for $19.5 \mathrm{~d}$ at lower sensitivity $(\epsilon=0.07$ ), while V2.0 operated for $9.4 \mathrm{~d}$ at the same sensitivity $(\epsilon=0.14)$. FRB survey V3.0 consists primarily of pointings taken commensally during pulsar timing observations. In this mode, the time series data from $352 \mathrm{FBs}$ are searched for dispersed single pulses in real time, using a custom version of the HEIMDALL software on 8 Nvidia GeForce GTX TITAN X (Maxwell) GPUs with a latency of 8-s. The resulting candidates were then processed offline, typically the following morning for overnight pulsar timing (RFI is much reduced at night, and the telescope is made available for maintenance on week days). On weekends, the telescope is usually operated continuously. The candidate processing pipeline used is described in detail in Caleb et al. (2016b). The process followed is:

(i) obtain 352 data streams (8-bits/sample), one for each FB, at 655.36- $\mu$ s sampling;

(ii) search time series for single pulses with width, $0.65536<W<41.943 \mathrm{~ms}\left(W=2^{\mathrm{N}} \times 0.65536 \mathrm{~ms}\right.$, where $N=0,1,2, \ldots)$ and DMs in the range $100<\mathrm{DM}<2000 \mathrm{pc} \mathrm{cm}^{-3}$;

(iii) remove events occurring simultaneously in more than three FBs at a given instant in time;

(iv) classify only events with $\mathrm{S} / \mathrm{N} \geq 10, \mathrm{DM} \geq 100 \mathrm{pc} \mathrm{cm}^{-3}$ and $W \leq 41.943 \mathrm{~ms}$ as potential FRB candidates. These then require human scrutiny of the diagnostic plots, to remove candidates that were RFI, almost always due to narrow-band mobile handset emissions in our operating passband and single pulses from known pulsars.

\section{RESULTS}

The false positive rate at UTMOST is high due to RFI caused by mobile phone handsets, which produce narrow band (5-MHz) emission in our band, typically in $\approx 20 \mathrm{~ms}$ pulses. These can be eliminated because celestial pulses are expected to be broad-band, modulated by a frequency dependent response across the $31.25 \mathrm{MHz}$ bandwidth. This process has been validated using individual pulses from about 20 bright pulsars seen to date. We are presently automating this process using machine learning algorithms, so that pulses can trigger a full voltage dump of the raw data while they are still in the $\approx 30$ s of ring buffer storage, with alerts issued in near real time. RFI occurs predominantly at low DM, but the rate is high enough to produce a few hundred spurious candidates above our DM limit of $100 \mathrm{pc} \mathrm{cm}^{-3}$ daily. Candidates were typically vetted each morning after data taking.

In 2016 March, April and June, we made the first interferometric detections of FRBs at 843 MHz: FRB 160317, FRB 160410 and FRB 160608, as shown in Fig. 1.

\subsection{FRB 160317}

This was detected on 2016 March 17 at 09:00:36.530 UTC while observing an X-ray magnetar SGR 0755-23, in response to an Astronomers Telegram (Barthelmy et al. 2016). The burst occurred about 0.4 east of the magnetar, and was detected $\sim 1^{\circ}$ off the Galactic plane with a DM of $1165(11) \mathrm{pc} \mathrm{cm}^{-3}$. The DM due to the ISM at this sight line is $\sim 320 \mathrm{pc} \mathrm{cm}^{-3}$ from the NE2001 model by Cordes \& Lazio (2002) and $~ 395 \mathrm{pc} \mathrm{cm}^{-3}$ from the YMW16 model (Yao, Manchester \& Wang 2017). The burst with S/N 13, occurred east of the centre of the primary FB of detection (Beam 212) since it appeared weakly in the adjacent FB with $\mathrm{S} / \mathrm{N} \sim 5$ (Beam 213) as shown in Fig. 2. 

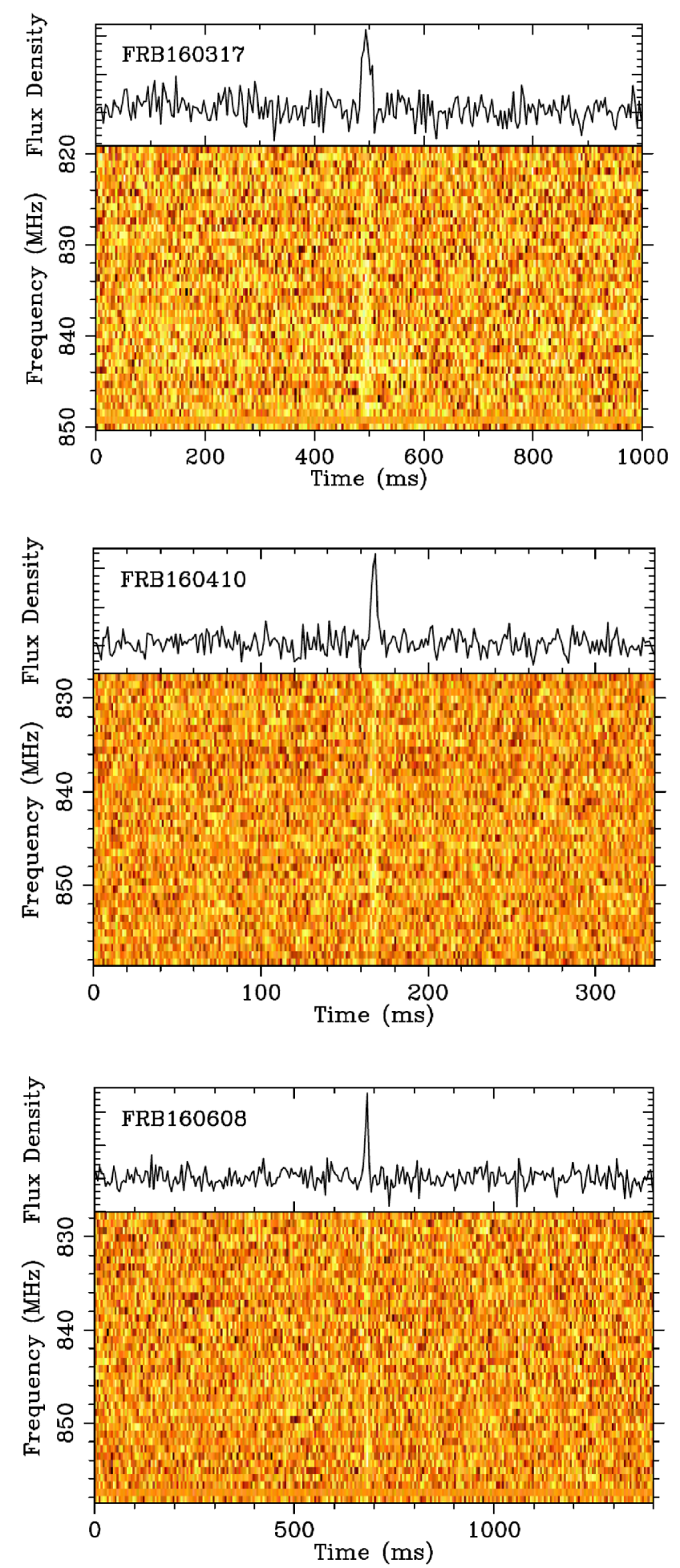

Figure 1. Frequency versus time behaviour of FRBs 160317, 160410 and 160608 detected at UTMOST at the centre frequency of $834.765 \mathrm{MHz}$. The top panel in each case shows the frequency-averaged pulse profile. The bottom panel shows that narrow-band RFI has been excised and the effects of interchannel dispersion have been removed assuming DMs of $1165 \pm 11$, $278 \pm 3$ and $682 \pm 7 \mathrm{pc} \mathrm{cm}^{-3}$, respectively. The data are uncalibrated as the bandpass of the system varies as a function of the meridian angle, and the flux densities are in arbitrary units. Note the different time range on the abscissa for FRB 160410.
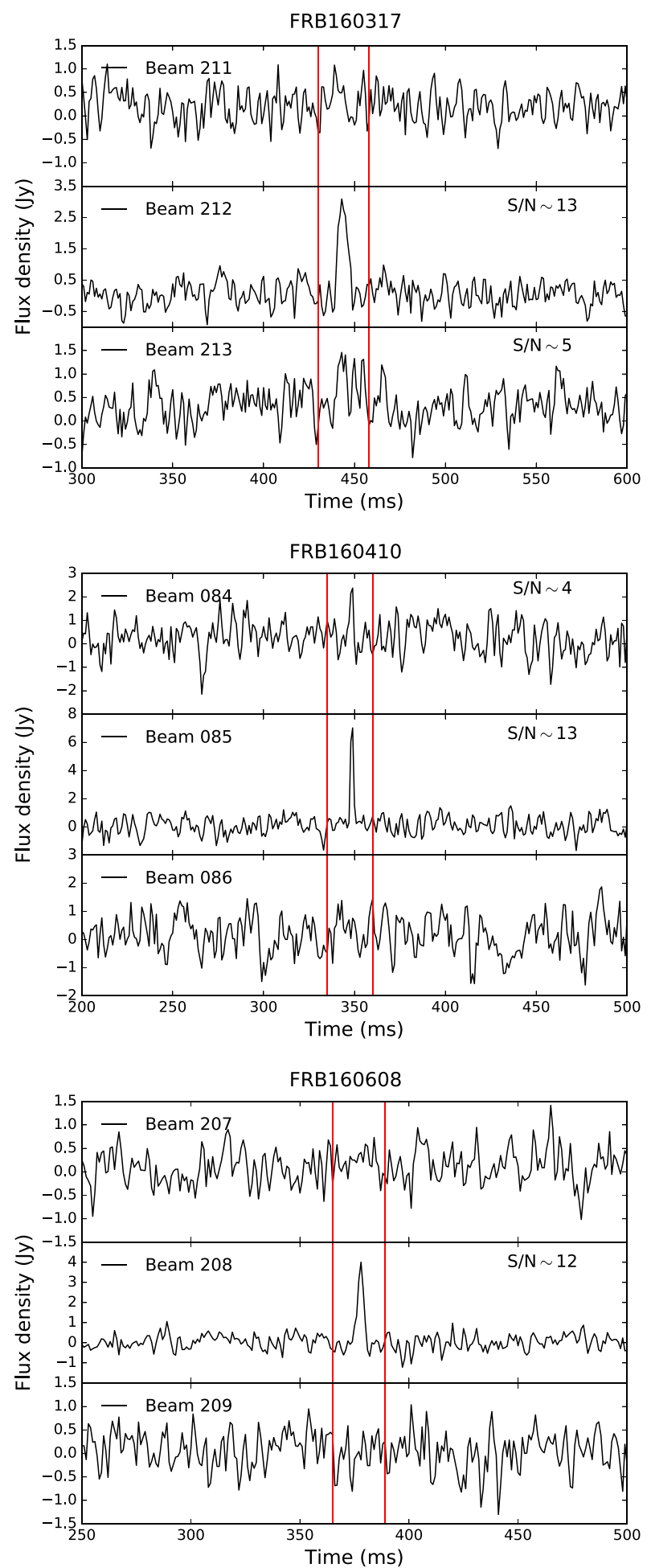

Figure 2. The three panels display the total power pulse profiles for one polarization in three adjacent FBs. FRBs 160317 and 160410 were also detected as sub-threshold events in neighbouring FBs (in addition to the high S/Ns in the primary detection FBs), indicating that they did not occur near the centres of the primary FB. On the contrary, FRB 160608 was only detected in one FB suggesting that it occurred close to the centre of beam 208 (see bottom panel). 


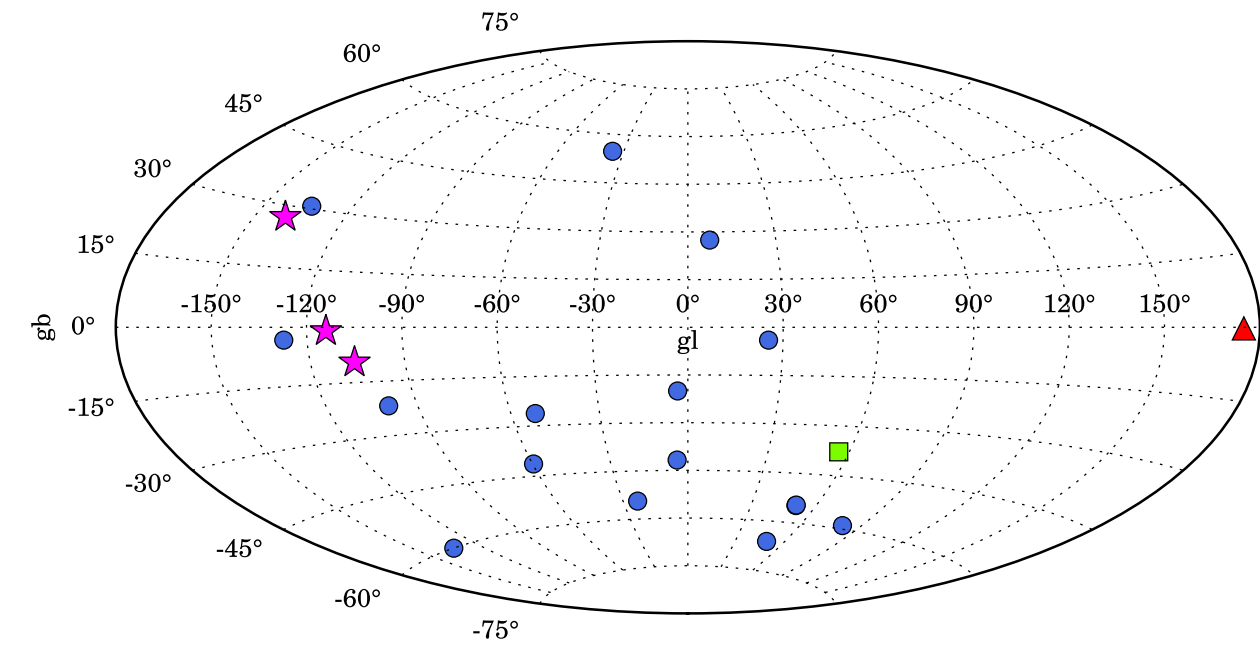

\begin{tabular}{|lllllllll|}
\hline$\circ$ & Parkes FRBs & $\downarrow$ & UTMOST FRBs & $\square$ & GBT FRB & & $\Delta$ & Arecibo FRB \\
\hline
\end{tabular}

Figure 3. The sky distribution of the 18 FRBs published to date in Galactic coordinates. Dots mark the positions of the FRBs detected at the Parkes telescope, the triangle represents FRB 121102 detected at the Arecibo telescope and the square represents FRB 110523 discovered at the GBT. Stars mark the positions of the UTMOST FRBs. Two of the Parkes FRBs have positions separated by 9 arcmin which are not resolved in this figure. It should be noted that there are large biases in this distribution due to very different sky coverages and survey depths.

\subsection{FRB 160410}

Similar to FRB 160317, this FRB was also detected in two adjacent FBs (Beam 085 with $\mathrm{S} / \mathrm{N} \sim 13$ and Beam 084 with $\mathrm{S} / \mathrm{N} \sim 4$ ) as seen in Fig. 2. A single dispersed pulse was discovered on 2016 April 04 at 08:33:39.680 UTC, in an observation of the pulsar J0837+0410 at the telescope's boresight. This pulsar is so bright that individual pulses were seen from it as the FRB occurred, meaning the flux density scale and bandpass response of the observation were well understood. The FRB was seen $\sim 1^{\circ}$ away from boresight. This pulse was detected at Galactic latitude, $\sim 27^{\circ}$ with the line-of-sight DM accounting for only $\sim 58 \mathrm{pc} \mathrm{cm}^{-3}$ of the total observed DM from the NE2001 model. The YMW16 model estimates $\sim 63 \mathrm{pc} \mathrm{cm}^{-3}$. FRB 160410 has one of the lowest DM excess' $\sim 220 \mathrm{pc} \mathrm{cm}^{-3}$ till date making it one of the closest known FRBs and an excellent candidate to search for repeat pulses.

\subsection{FRB 160608}

The burst occurred in an observation of the pulsar J0738-4042 at $l=254.11$ and $b=-9.54$ on 2016 June 06 at 03:53:01.088 ut with a total DM of $\sim 682 \mathrm{pc} \mathrm{cm}^{-3}$ and $\sim 238 \mathrm{pc} \mathrm{cm}^{-3}$ contribution from the Milky Way (NE2001). The YMW16 model's estimate however is $\sim 310 \mathrm{pc} \mathrm{cm}^{-3}$. It was seen $\sim 0.5$ from the boresight position. FRB 160608 was detected with $\mathrm{S} / \mathrm{N} \sim 12$, just above the detection threshold of 10 and it occurred towards the centre of the primary detection FB (Beam 208). No pulse was detected in the adjacent FBs (see Fig. 2). This was initially of concern, but tests with the Vela pulsar placed sufficiently far south of the telescope boresight, to produce an individual pulse with the same $\mathrm{S} / \mathrm{N}$ showed that detection in a single FB occurred $\approx 20$ per cent of the time. The localization of this FRB is thus slightly poorer $(21 \operatorname{arcsec} \times 8.4)$ than for the other two FRBs, for which a two FB detection allow a more accurate position.

The sky distribution of the 3 FRBs in Galactic coordinates, with respect to the positions of other published FRBs is shown in Fig. 3. All 3 FRBs have been localized to narrow ellipses on the sky with their orientation hour angle dependent as seen in Fig. 4. The primary advantage of the array is that a pulse from a far-field point source is detected in a maximum of three adjacent FBs at any given time, confirmed by extensive pulsar observations. RFI is typically nearfield, and predominantly appears in more than three adjacent FBs, meaning that it can be reliably excised to reduce false positive rates when searching for transients. Using the adjacent FB detections of FRB 160317, we have modelled the point of separation between the near-field or Fresnel region and the far-field or Fraunhofer region of the telescope. Assuming a point source at $10^{6} \mathrm{~km}$, we compute the S/N for a tied-array beam (e.g. FB 212) phased at an offset of 0.3 from the centre of the beam to ensure a two FB detection. We compute the path length to each module, the phase of the signal along the array and perpendicular to the array, and add all these as a vector sum weighted by the module performance, to get the 'boresight' S/N. We see that in Fig. 5 at a distance of $\gtrsim 10^{4} \mathrm{~km}$, we achieve a two $\mathrm{FB}$ detection with $\mathrm{S} / \mathrm{N} \sim 13$ in the primary detection beam and $\mathrm{S} / \mathrm{N} \sim 5$ in the secondary detection beam, similar to the FRB being modelled. Detections of FRBs in one or two FBs only, thus allows us to identify them as sources more distant than this, placing them well away from the Earth and hence effectively rule out sources of local origin.

The discovery observations containing the FRBs were carefully inspected to check for similar events at the same time and with the same DM as the FRB, in other FBs. No other broad-band pulses were detected in any other FBs within approximately $60 \mathrm{~s}$ of the bursts. Moreover, in addition to all the tied array FBs, we form a single special FB as the incoherent sum of all the other FBs. This 'total power' FB was also searched for events near the UTC of the three bursts. For the three FRBs, this FB contained no unusual sources of RFI. Only twice during the three surveys did we find FRB-like candidates (i.e. appearing across the band) that were identified as RFI upon closer analysis. In each case, similar events could be found in dozens to hundreds of FBs, and were thus obvious near-field RFI. These false candidates also had 'patchy' power across the observing band, indicative of RFI 

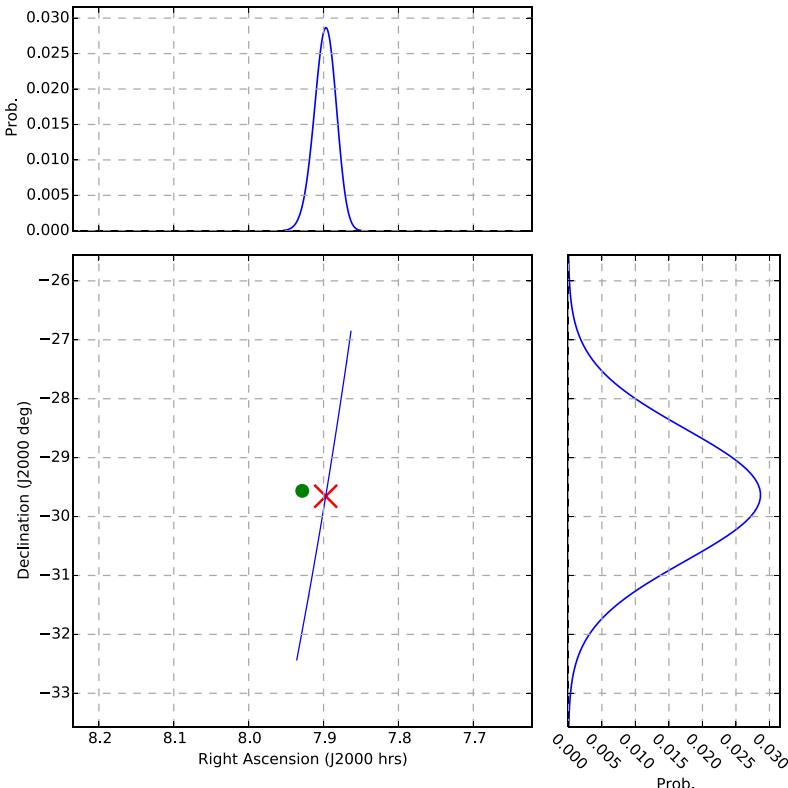
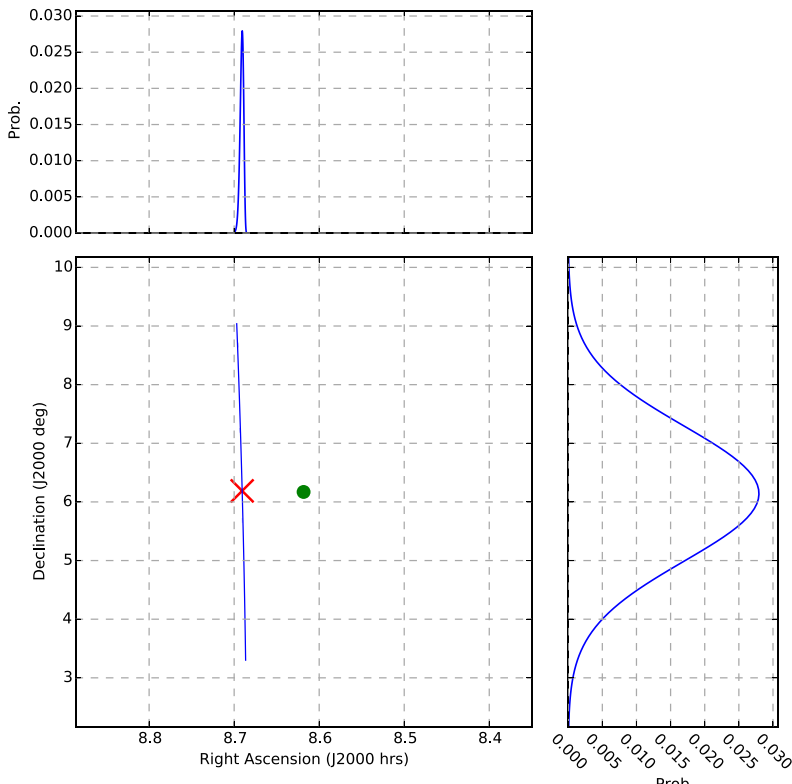
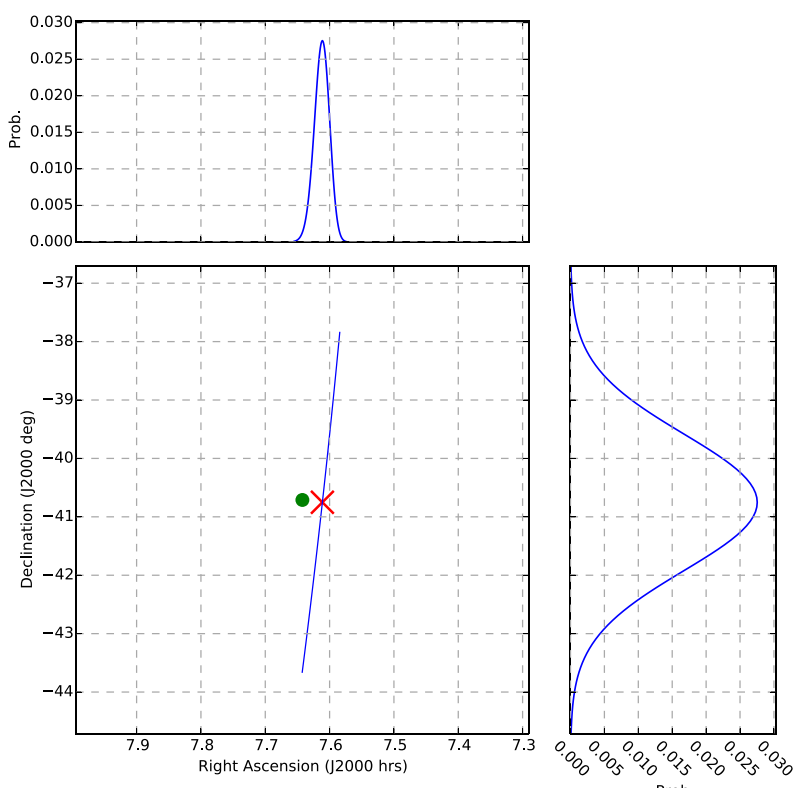

Figure 4. We show $3 \sigma\left(15 \operatorname{arcsec} \times 8^{\circ} 4\right)$ localization ellipses of FRB 160317 (top-left), FRB 160410 (top-right) and FRB 160608 (bottom-centre). The maximum probability in RA (J2000) and DEC (J2000) assuming a Gaussian probability density function gives the most likely position of the FRB, and is marked by the cross. The dot indicates the position of the 'boresight' pointing of the telescope. Typically, a pulsar is being timed at this position in the telescope beam. In two out of three cases, the pulsar at this position was bright enough to see individual pulses during the observation when an FRB was detected. The coordinates of the ellipses are given in Table A1.

generated from different carrier handsets operating at the same time in our band.

Two of the three FRBs have been discovered relatively close to the Galactic plane, with the locations marked as stars in Fig. 3. All three have DMs significantly in excess of the Galactic contribution, suggesting an extragalactic or cosmological origin. Under this assumption, the contribution from the IGM to the DM can be used to infer a redshift, using the scaling relation in Ioka (2003) and Inoue (2004). This places FRBs 160317, 160410 and 160608 at a redshift upper limit of $0.7,0.2$ and 0.4 , respectively, assuming zero contribution from any potential host galaxy. Any contribution from a host galaxy or the immediate vicinity of an associated source, could be a significant fraction of the total DM depending on its orientation and location. The average DM for elliptical galaxies is $37 \mathrm{pc} \mathrm{cm}^{-3}$ and for spiral galaxies is $45 \mathrm{pc} \mathrm{cm}^{-3}$ based on the probability distribution of DMs computed for a range of host galaxies (Xu \& Han 2015). For spirals, the weighted average over a range of inclination angles is estimated to be $142 \mathrm{pc} \mathrm{cm}^{-3}$. However, the host contribution to the DM from high-redshift galaxies can be small due to cosmological time dilation and the corresponding redshifting of frequency (Zhou et al. 2014). It also does not account for any bias in the FRB locations within galaxies. 


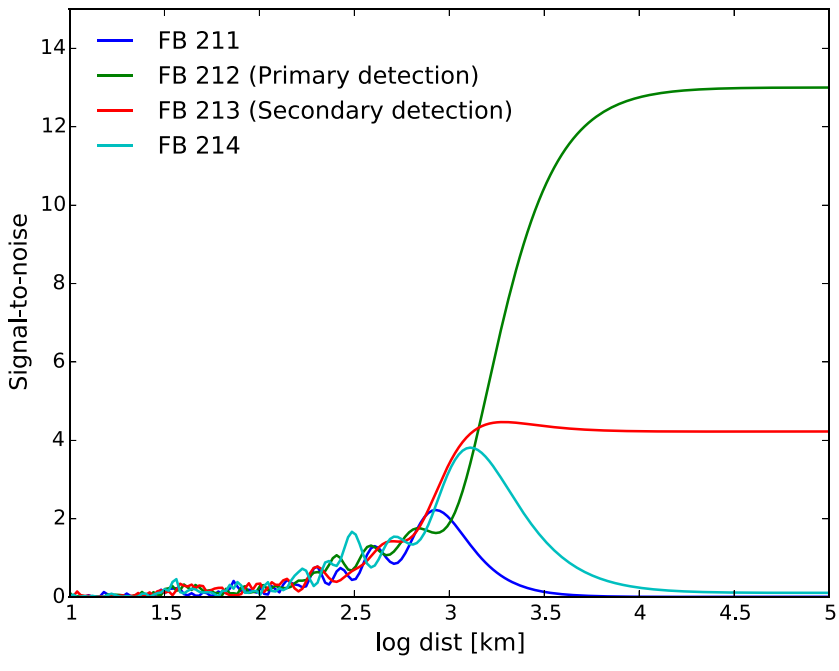

Figure 5. Simulations of the detections of FRB 160317 in adjacent FBs to determine the Fresnel limit of the telescope. At a distance of $\gtrsim 10^{4} \mathrm{~km}$, the S/Ns of the modelled pulse in FB 212 and FB 213, match that of the observations with non-detections in the other FBs.

The S/Ns, DMs and widths of all three FRBs have been computed using the DESTROY single pulse search software, PSRCHIVE, with scripts made publicly available through the FRBCAT repository. The observed widths of all three FRBs are dominated by dispersion smearing as shown in Table 1 . This is due to our small bandwidth and limited number of channels (40 channels). We have now implemented a fine channel mode (320 channels) that will potentially increase our sensitivity and the FRB detection rate by a factor of $\sqrt{8}$. Our total bandwidth of only $31.25 \mathrm{MHz}$ is too narrow to permit a measurement of dispersion index. Single pulses from the Vela pulsar were used to test our sensitivity to the DM index. The DM and the DM index $\delta$ where the dispersive delay is given by

$\Delta t \propto \Delta v^{-\delta}$

are found to be highly correlated, so that we can place no practical limit on $\delta$. We therefore set the DM index to $\delta=-2$.

\subsection{Localization}

All three FRBs were discovered while following up known sources, which were at 'bore-sight', and thus centred on Beam 177. We localize each FRBs position in the sky, using the angular separation between the FRB's position in FB space from the bore-sight FB (see Fig. 5). The $1 \sigma$ uncertainty in the direction of the semimajor axis is defined by the primary beam $(\sim 2.8)$ while the uncertainty in the semiminor axis is controlled by the fractional $\mathrm{S} / \mathrm{N}$ measured from the adjacent FB detections. For FRBs detected in adjacent FBs, we perform a simple linear interpolation based on the $\mathrm{S} / \mathrm{N}$ to localize the event in the FB grid. For the FRB with a single FB detection only, we assume the centre of the FB for the localization. This allows us to construct a trace on the sky relative to the bore-sight (RAJ, DECJ), taking into account the meridian angle of the observation at the time of the FRB, and the known, slight skew and tilt of the east-west

Table 1. Table of observed and inferred properties of the three FRBs in this work. The UTCs are the start times of the observations and the times at which the events occurred. Sky coordinates are the most likely positions of the FRB event within a narrow error ellipse (see Fig. 4). The peak fluxes ( $S_{\text {peak, obs }}$ ) are computed using the radiometer equation and the DM contribution from the Milky Way $\left(\mathrm{DM}_{\mathrm{Gal}}\right)$ is calculated using the NE2001 model (Cordes \& Lazio 2002). The 'boresight fluence' is the detected fluence corrected for the primary beam and FB responses. They are shown as lower limits, for the unknown correction to higher fluence along the semimajor axis of the detection FB. The isotropic energy $E_{0}$ is the energy at source and $\tau 843 \mathrm{MHz}$ is the DM smearing due to the pulse broadening caused by the incoherent dedispersion at the observing frequency. The observed widths and their uncertainties are measured using the DESTROY (https://github.com/evanocathain/destroy_gutted) single pulse search software, PSRCHIVE (http://psrchive.sourceforge.net/) and scripts made publicly available through the FRBCAT repository (https://github.com/frbcat/FRBCAT_analysis). The redshift $z$ is computed as $\left(\mathrm{DM}_{\mathrm{FRB}}-\mathrm{DM}_{\mathrm{Gal}}\right.$, NE2001)/1200 (Ioka 2003; Inoue 2004). The luminosity and comoving distances are calculated for a standard, flat-universe $\Lambda$ CDM cosmology using COSMOCALC (Wright 2006). The boresight sources are the magnetar or pulsars that were being observed during the time the FRB occurred.

\begin{tabular}{|c|c|c|c|}
\hline Parameter & FRB 160317 & FRB 160410 & FRB 160608 \\
\hline UTC start & 2016-03-17-08:30:58 & 2016-04-10-08:16:54 & 2016-06-08-03:52:24 \\
\hline UTC event & 2016-03-17-09:00:36.530 & 2016-04-10-08:33:39.680 & 2016-06-08-03:53:01.088 \\
\hline RA J2000 (hh:mm:ss) & $07: 53: 47$ & $08: 41: 25$ & $07: 36: 42$ \\
\hline DEC J2000 (dd:mm:ss) & $-29: 36: 31$ & $+06: 05: 05$ & $-40: 47: 52$ \\
\hline$l\left(^{\circ}\right)$ & 246.05 & 220.36 & 254.11 \\
\hline$b\left(^{\circ}\right)$ & -0.99 & 27.19 & -9.54 \\
\hline Detection $\mathrm{S} / \mathrm{N}$ & 13 & 13 & 12 \\
\hline$S_{\text {peak, obs }}(\mathrm{Jy})$ & $>3.0$ & $>7.0$ & $>4.3$ \\
\hline Boresight fluence (Jy ms) & $>69$ & $>34$ & $>37$ \\
\hline Isotropic energy, $E_{0}(\mathrm{~J})$ & $\sim 10^{34}$ & $\sim 10^{32}$ & $\sim 10^{33}$ \\
\hline Observed width, $W(\mathrm{~ms})$ & $21(7)$ & $4(1)$ & $9(6)$ \\
\hline DM smearing, ${ } 843 \mathrm{MHz}(\mathrm{ms})$ & 12.6 & 3.0 & 7.4 \\
\hline $\mathrm{DM}_{\mathrm{FRB}}\left(\mathrm{pc} \mathrm{cm}^{-3}\right)$ & $1165(11)$ & $278(3)$ & $682(7)$ \\
\hline $\mathrm{DM}_{\mathrm{Gal}, \mathrm{NE} 2001}\left(\mathrm{pc} \mathrm{cm}^{-3}\right)$ & 319.6 & 57.7 & 238.3 \\
\hline $\mathrm{DM}_{\mathrm{Gal}}, \mathrm{YMW} 16\left(\mathrm{pc} \mathrm{cm}^{-3}\right)$ & 394.6 & 62.5 & 310.3 \\
\hline Inferred redshift, $z$ & 0.7 & 0.2 & 0.4 \\
\hline Luminosity distance (Gpc) & 4.30 & 0.89 & 1.97 \\
\hline Comoving distance $(\mathrm{Gpc})$ & 2.52 & 0.75 & 1.44 \\
\hline Boresight source & SGR 0755-2933 & $\mathrm{J} 0837+0610$ & J0738-4042 \\
\hline
\end{tabular}



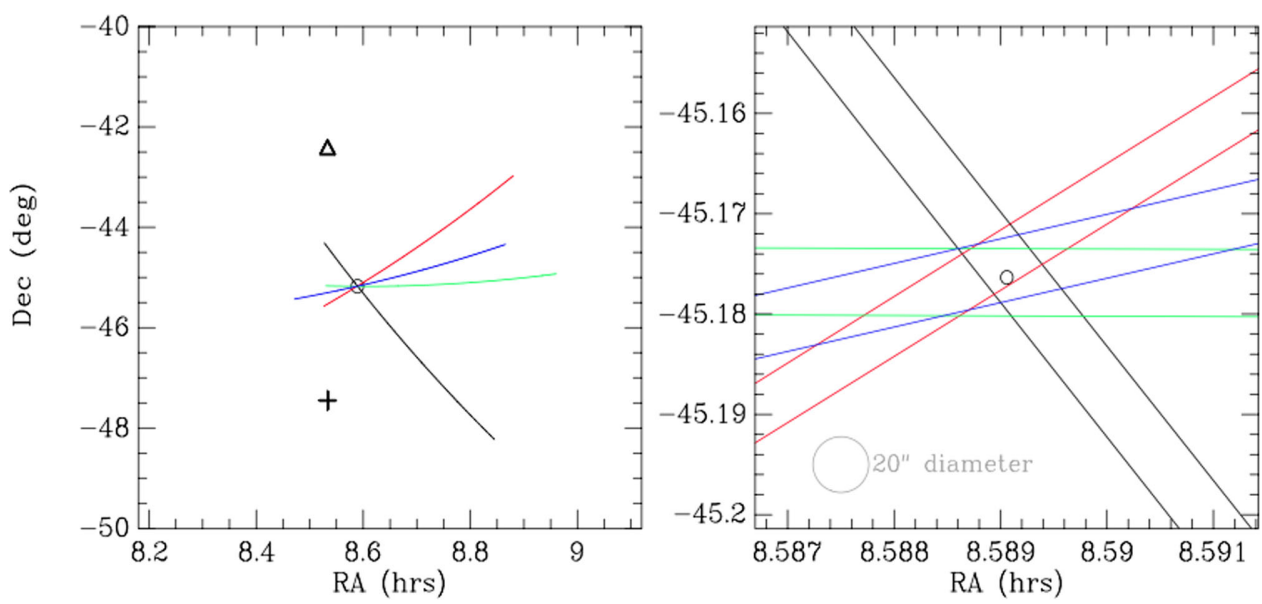

Figure 6. Left-hand panel: localization contours for four single pulses from Vela, observed over different hour angles and distances from the telescope boresight. The triangle marks the boresight position for the black FB and the cross marks the boresight position for the three other FBs. Right-hand panel: a zoom into the $3 \sigma$ error ellipse for the position of the source on the sky. The circle indicates the position of the pulsar. Even a single repeat of an FRB at a different hour angle, could constrain the position to a few arcsec radius shown in localization contours in the right-hand panel.

arms relative to true east-west and horizontal. The trace is a strong function of the hour angle of the observation, as one would expect in an east-west array. The uncertainty in the direction of the semiminor axis was confirmed observationally using single pulse detections from bright pulsars. Single pulses that were detected in two adjacent FBs with S/N similar to our FRBs were chosen to estimate our localization accuracy on the sky. These FB localizations were then compared to the true position of the pulsar. The $1 \sigma$ scatter of the calculated position of the pulsar from individual pulses, compared to the known position of the pulsar, is $\sim 0.1 \mathrm{FBs}$ or $\sim 5 \mathrm{arcsec}$, in the direction perpendicular to the FB. For single FB detections at low $\mathrm{S} / \mathrm{N}$, a similar analysis yielded a slightly poorer localization precision of 7 arcsec. Two of the FRBs found with UTMOST have FWHP error ellipses of $5 \operatorname{arcsec} \times 2.8\left(\sim 11 \operatorname{arcmin}^{2}\right)$ on the sky as seen in Fig. 4. For comparison, single-beam FRB detections (with FWHP beamwidth of 14.0 arcsec) at Parkes are localized to $\sim 160 \operatorname{arcmin}^{2}$. The probability density of the localization is shown in right ascension (RA) and declination (DEC), with the cross marking the most likely position of the burst for each FRB.

One of the advantages of UTMOST is that we can localize pulses to a few arcsec radius if the source is observed at different hour angles. The tilt of the error ellipses seen in Fig. 4 demonstrates this and is a result of the geometry of the telescope. The most likely position of the FRB is marked by the cross. The FBs rotate in position according to the hour angle pointing of the telescope. A repeat FRB pulse, at a significantly different hour angle, allows us to localize the bursts to a few arcsec, depending on the $\mathrm{S} / \mathrm{N}$ of the event. We have tested this scenario using individual pulses from four different pulsars, with results for four different hour angles (and offsets from boresight) for the Vela pulsar shown in Fig. 6. Localizations to about 5 arcsec accuracy, or 0.1 FB widths, should be possible for a bright, repeating FRB. UTMOST is part of the shadowing campaign of the Survey for Pulsars and Extragalactic Radio Bursts (SUPERB) at the Parkes radio telescope (Keane et al., in preparation). An FRB detected simultaneously with Parkes and UTMOST will yield a good localization even if the burst does not repeat. The sky positions of the three FRBs were also re-observed at different HAs, to look for additional bursts. We spent $105 \mathrm{~h}$ following FRB 160317, $43 \mathrm{~h}$ on FRB 160410 and $35 \mathrm{~h}$ on FRB 160608. The data were searched offline for pulses with $\mathrm{S} / \mathrm{N} \geq 10$ and with \pm 20 per cent of the DM of the FRB, using the pipeline described in Section 2. No repeat pulses were found from any of the FRB positions.

\section{FRB EVENT RATE AT UTMOST}

\subsection{Event rate analysis}

Connor et al. (2016b) present detailed analyses constraining the FRB rates at various telescopes, scaling from a single FRB discovered at $800 \mathrm{MHz}$ at the GBT (FRB 110523) (Masui et al. 2015). They estimate a rate of $4.2_{-3.2}^{+19.6} \times 10^{-1} \mathrm{~d}^{-1}$ at UTMOST operating at its design sensitivity, based on comparisons between the sensitivities and areas surveyed by the two telescopes. This estimate is consistent with Caleb et al. (2016b). Using the same method as outlined in Connor et al. (2016b), we calculate a rate of $0.014_{-0.013}^{+0.05} \mathrm{~d}^{-1}$, which agrees with the rate we measure at UTMOST. We have spent a total of $180 \mathrm{~d}$ on sky and discovered three FRBs with an FoV of $8.8 \mathrm{deg}^{2}$. Based on this, we measure a detectable event rate of $(\mathcal{R})$,

$\mathcal{R}(\mathcal{F} \gtrsim 11 \mathrm{Jy} \mathrm{ms}) \gtrsim 0.78_{-0.57}^{+1.24} \times 10^{2}$ events $\mathrm{sky}^{-1} \mathrm{~d}^{-1}$

at the 95 per cent confidence level (Gehrels 1986), above a full power boresight fluence of $11 \mathrm{Jy} \mathrm{ms}$ as parametrized by equation (2), at the half-power FoV. The rate is given as a lower limit since all searches are incomplete in the fluence-width plane. Following Keane \& Petroff (2015), our fluence complete rate is

$\mathcal{R}(\mathcal{F} \gtrsim 69 \mathrm{Jy} \mathrm{ms}) \sim 5.0_{-4.7}^{+18.7}$ events sky ${ }^{-1} \mathrm{~d}^{-1}$,

as shown in Fig. 7.

In Caleb et al. (2016a,b), we have made estimates of the event rate expected at UTMOST, scaling from the event rate at Parkes, under assumptions about the integral source count distribution $(\log N-$ $\log \mathcal{F}$ relation) and the spectral index of FRBs. To do this, we assume that the spectral energy distribution (SED) is flat between the Parkes 1.4 GHz and UTMOST's $843 \mathrm{MHz}$ operating frequencies, and that the source count distribution scales as

$N\left(>\mathcal{F}_{\text {lim }}\right)=\mathcal{F}_{\text {lim }}^{\alpha}$

where $\alpha=-3 / 2$ for events populated in a Euclidean Universe. Under these assumptions, Caleb et al. (2016b) predict a rate of $\gtrsim 0.008(0.004)$ events $\mathrm{d}^{-1}$ for a $10 \sigma, 1$-ms wide FRB to a minimum 


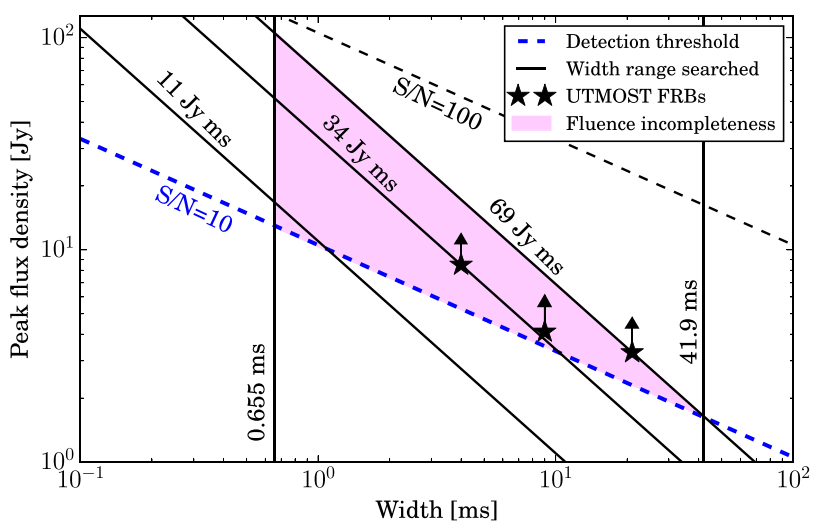

Figure 7. Boresight corrected peak flux density versus observed width for the three FRBs. Dashed lines represent lines of constant $\mathrm{S} / \mathrm{N}$ and solid lines represent lines of constant fluence. The range of widths searched is enclosed by the solid vertical lines. The pink region is the fluence incomplete region that indicates that pulses with the same fluence but different widths are not equally detectable. Only pulses above $69 \mathrm{Jy} \mathrm{ms}$ are detectable across the entire width range searched at UTMOST.

detectable fluence of $\mathcal{F}_{\text {lim }}=11 \mathrm{Jy}$ ms at boresight (see equation 2). If we correct this rate for the measured primary beam dimensions being 10 per cent larger (Bailes et al., submitted) than adopted in Caleb et al. (2016b) we get a rate of $\gtrsim 0.007(0.004)$ events $d^{-1}$ that translates to being able to detect 1.3 events in $180 \mathrm{~d}$ on sky. This is in mild tension with our discovery of three events in the survey. We quantify this tension by calculating the probability of observing three or more events to be 14.3 per cent, assuming Poisson statistics with a mean of 1.3. Note that the predicted rate at UTMOST takes into account pulse-width broadening in the current implementation of the back end (channel widths $\sim 780 \mathrm{kHz}$ ) but does not account for possibly highly atypical scintillation properties along specific lines of sight to FRB events. The difference in the estimated and measured rates could be due to FRBs being brighter than expected at $843 \mathrm{MHz}$, and/or the slope of the source count distribution $\alpha$, being shallower than the assumed value. Simultaneous broad-band detection of an FRB (e.g. Parkes + UTMOST) would help constrain the spectral index and resolve the question. In Caleb et al. (2016a), we measured $\alpha \approx-0.9 \pm 0.3$, from nine FRBs discovered in the high latitude sub-survey of the HTRU survey at Parkes. This is consistent with the events occurring at cosmological distances in a $\Lambda \mathrm{CDM}$ Universe, in which the Euclidean value for $\alpha$ does not hold. Assuming a flat spectral index for FRBs $(\gamma=0)$, if we scale the rate at UTMOST from equation (4), assuming $\alpha=-1.0$ for the slope of the $\log N-\log \mathcal{F}$ relation based on the best fit from the bottom panel in Fig. 8, we obtain a rate of $\gtrsim 2.1 \times 10^{3}$ events sky ${ }^{-1} \mathrm{~d}^{-1}$ at $1.4 \mathrm{GHz}$, consistent with the observed Parkes rate at the $2 \sigma$ level (Champion et al. 2016).

\subsection{Constraints on spectral and source count distribution indices}

As discussed in the previous section, the observed FRB rate at UTMOST at $843 \mathrm{MHz}$ can be brought to consistency with the rate found at $1.4 \mathrm{GHz}$ at Parkes if we assume FRBs are flat spectrum sources on average, and that the $\log N-\log \mathcal{F}$ relation has slope $\alpha=-1.0$ (best fit for $\alpha$ assuming $\gamma=0$, in bottom panel of Fig. 8). Alternatively, we can relax the flat spectrum assumption, and ascribe the higher than expected rate to FRBs being brighter at $843 \mathrm{MHz}$ than at $1.4 \mathrm{GHz}$. Assuming the $\log N-\log \mathcal{F}$ relation has slope $\alpha$ and that the FRBs have a power-law SED with index $\gamma$ such that $S \propto$ $v^{\gamma}$, we examine the following scenarios.

(i) $\alpha=-3 / 2$ : based on the detection rates at the Parkes, GBT and UTMOST telescopes, we can constrain a spectral index for FRBs as shown in Fig. 8. The rate of $\sim 7000$ events $\mathrm{sky}^{-1} \mathrm{~d}^{-1}$ at $1.4 \mathrm{GHz}$ at Parkes, above a fluence limit of $0.4 \mathrm{Jy} \mathrm{ms}$ (Champion et al. 2016) scales to $\sim 56$ events sky ${ }^{-1} \mathrm{~d}^{-1}$ above UTMOST's fluence limit of $11 \mathrm{Jy} \mathrm{ms}$, and assuming the spectral index to be flat $(\gamma=0)$ (see Section 4.1). Similarly, the rate of $\sim 2.7 \times 10^{4}$ events sky ${ }^{-1} \mathrm{~d}^{-1}$ at $800 \mathrm{MHz}$ at the GBT, above a fluence threshold of $0.3 \mathrm{Jy} \mathrm{ms}$ (Connor et al. 2016b) and scaled to UTMOST's fluence threshold of $11 \mathrm{Jy} \mathrm{ms}$ is $\sim 116$ events $\mathrm{sky}^{-1} \mathrm{~d}^{-1}$. Using these values, we fit for the FRB spectra to be $\gamma=-1.1(1.2)$ (top panel of Fig. 8). This value is found to be consistent with most of the estimates for

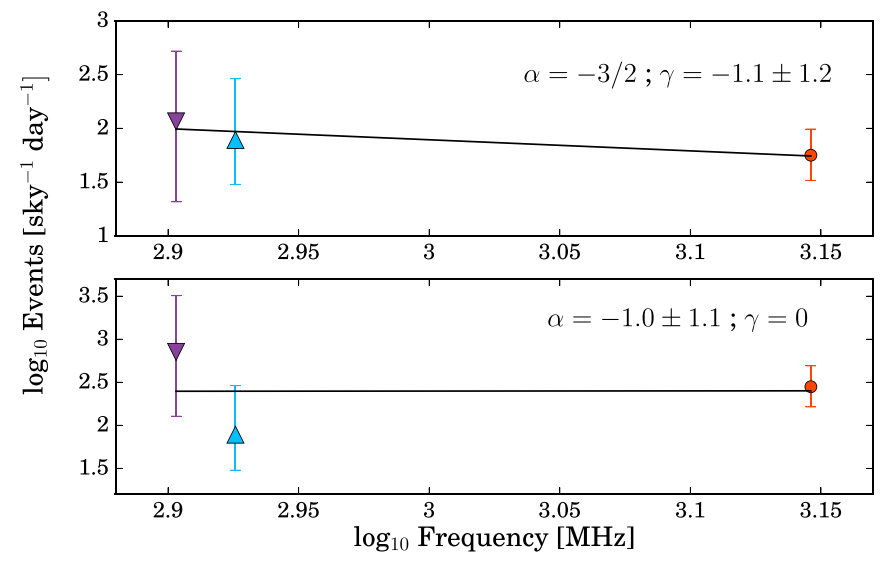

$-\bar{\gamma}$ Rate of 27000 events above $0.3 \mathrm{Jy} \mathrm{ms}$ at $800 \mathrm{MHz}$ (Connor et al. 2016b) scaled to $11 \mathrm{Jy} \mathrm{ms}$
$\overline{-}$ Rate of 78 events $\geq 11 \mathrm{Jy} \mathrm{ms}$ at $843 \mathrm{MHz}$ (This work)
- - Rate of 7000 events above $0.4 \mathrm{Jy} \mathrm{ms}$ at $1.4 \mathrm{GHz}$ (Champion et al. 2016) scaled to $11 \mathrm{Jy} \mathrm{ms}$

Figure 8. All-sky rates at the GBT and Parkes telescope, scaled to the measured UTMOST fluence limit of 11 Jy ms. For events to be detectable at UTMOST's present sensitivity, either (1) the spectral index should be steep or (2) the slope of $\log N-\log \mathcal{F}$ should be flat. Top panel: constraint on $\gamma$ assuming $\alpha=-3 / 2$. Bottom panel: best fit for $\alpha$ assuming $\gamma=0$. 
UTMOST in Rajwade \& Lorimer (2017) for both the cosmological and extragalactic scenarios. It is also found to be in good agreement with the observed constraint in Keane et al. (2016) albeit for just one FRB.

(ii) $\gamma=0$ : a similar analysis can be done to constrain the index $\alpha$ of the integral source count distribution assuming a flat spectral index. We constrain a value of $\alpha=-1.0$ (1.1) for $\gamma=0$ (bottom panel of Fig. 8). This value of $\alpha$ gives scaled rates of $\sim 270$ events sky $^{-1} \mathrm{~d}^{-1}$ at $1.4 \mathrm{GHz}$ at Parkes and $\sim 690$ events $\mathrm{sky}^{-1} \mathrm{~d}^{-1}$ at $800 \mathrm{MHz}$ at GBT.

Another possible scenario is that FRBs are giant pulses from pulsars (Cordes \& Wasserman 2016). The average observed spectral index for pulsars is $\gamma=-1.6$ (Jankowski et al., in preparation). If we assume this to be typical of FRBs, we fit a slope of $\alpha=-1.76$ for their $\log N-\log \mathcal{F}$ distribution. We note that the repeat FRB pulses from the Arecibo FRB 121102 exhibit a wide range of spectral indices ( $\gamma \sim-10$ to +14 ; Spitler et al. 2016), similar to giant pulses from the Crab pulsar. For example, giant pulses from the Crab pulsar exhibit spectral volatility in their broad range of spectral indices ( $\gamma \sim-15$ to +10 ; Karuppusamy, Stappers \& van Straten 2010), therefore it will be difficult to estimate the mean of the spectral indices until the numbers are sufficiently high. From simultaneous observations of FRB 150418 with Parkes at $1.4 \mathrm{GHz}$ and the Murchison Widefield Array (MWA) at $150 \mathrm{MHz}$ (Keane et al. 2016), the non-detection at the MWA places a limit of $\gamma>-3.0$. BurkeSpolaor et al. (2016) estimate a weak constraint of $-7.6<\gamma<5.8$ based on the detection sensitivity of Champion et al. (2016) which is consistent with our estimated values. These constraints are only valid if the SED is an unbroken broad-band power law and insignificantly affected by scintillation. This remains to be observationally proven. Future broad-band instruments like CHIME (Bandura et al. 2014) should have high FRB discovery rates and spectral coverage to definitively test this. Using the method in Caleb et al. (2016b), we scale the observed rate at UTMOST for a boresight fluence of $11 \mathrm{Jy} \mathrm{ms}$, to estimate the rates at CHIME and HIRAX (Newburgh et al. 2016) under a Euclidean Universe assumption. We expect CHIME to detect $\sim 70$ events beam ${ }^{-1} \mathrm{~d}^{-1}$ for $T_{\text {sys }}=50 \mathrm{~K}$, $\mathrm{S} / \mathrm{N}=10, G=1.38 \mathrm{~K} \mathrm{Jy}^{-1}, N_{\mathrm{p}}=2$ and $\mathrm{FoV}=250 \mathrm{deg}^{2}$ (Connor et al. 2016a; $\mathrm{Ng}$ et al. 2017). Similarly, we expect 350 events beam ${ }^{-1} \mathrm{~d}^{-1}$ at HIRAX for $T_{\text {sys }}=50 \mathrm{~K}, \mathrm{~S} / \mathrm{N}=10, G=10.5 \mathrm{~K} \mathrm{Jy}^{-1}$, $N_{\mathrm{p}}=2$ and FoV $=56 \mathrm{deg}^{2}$ (Newburgh et al. 2016).

\section{CONCLUSIONS}

In this paper, we present the first interferometric detections of FRBs, found during $180 \mathrm{~d}$ on sky at UTMOST. The events are beyond the $\approx 10^{4} \mathrm{~km}$ near-field limit of the telescope, ruling out local (terrestrial) sources of interference as a possible origin. We demonstrate with pulsars that a repeating FRB seen at UTMOST has the potential to be localized to $\approx 15$ arcsec diameter error circle, an exciting prospect for identifying the host.

An all-sky rate of $\mathcal{R}(\gtrsim 11$ Jy $\mathrm{ms}) \quad \gtrsim 0.78_{-0.57}^{+1.24} \times$ $10^{2}$ events $\mathrm{sky}^{-1} \mathrm{~d}^{-1}$ at $843 \mathrm{MHz}$ is calculated from our three events, at the boresight fluence out to the half-power FoV. Based on the time spent on sky and the number of detections made, we measure a rate of $0.017_{-0.01}^{+0.03}$ events beam ${ }^{-1} \mathrm{~d}^{-1}$ at UTMOST, for the sensitivity achieved during the upgrade. The rates estimated by Caleb et al. (2016b) for the present sensitivity, is only 14.3 per cent unlikely assuming Poisson statistics with a mean of 1.3. One possibility could be due to the $\log N-\log \mathcal{F}$ relation for events being flatter than for a Euclidean Universe, having a slope $\alpha \approx-1.0$, rather than $\alpha=-3 / 2$. In this case, searching for FRBs with a less sensitive, but wider field of view instrument, appears to be a competitive strategy, (e.g. Vedantham et al. 2016). Alternatively, FRBs may simply be brighter at $843 \mathrm{MHz}$ on average than at $1.4 \mathrm{GHz}$, implying a steeper spectral index for FRBs. Assuming a Euclidean Universe scaling, we find a best-fitting spectral index of $\gamma=-1.1(1.2)$. Our ongoing work, and the work of others at many other facilities, will settle these questions once sufficient numbers of FRBs are detected over a broad frequency range. Understanding the spectra and $\log N-\log \mathcal{F}$ distributions are vital in the quest to understand this enigmatic population.

\section{ACKNOWLEDGEMENTS}

The authors would like to thank the referee for their insightful suggestions. We would also like to thank Jr-Wei Tsai and Liam Connor for useful discussions. The Molonglo Observatory is owned and operated by the University of Sydney with support from the School of Physics. The upgrade to the observatory has been supported by the University of Sydney through the Federation Fellowship FF0561298 and the Science Leveraging Fund of the New South Wales Department of Trade and Investment. Support for operations and continuing development of the observatory is provided by the University of Sydney, Swinburne University of Technology, the Australian Research Council Centre for All-Sky Astrophysics (CAASTRO), through project number CE110001020, and the Laureate Fellowship FL150100148. The late Professor George Collins allocated strategic funds for the purchase of the supercomputer in use at the facility from Swinburne University and was a passionate advocate for this project.

\section{REFERENCES}

Bandura K. et al., 2014, Proc. SPIE, 9145, 914522

Barthelmy S. D. et al., 2016, Astron. Telegram, 8831

Burke-Spolaor S. et al., 2016, ApJ, 826, 223

Caleb M., Flynn C., Bailes M., Barr E. D., Hunstead R. W., Keane E. F., Ravi V., van Straten W., 2016a, MNRAS, 458, 708

Caleb M. et al., 2016b, MNRAS, 458, 718

Champion D. J. et al., 2016, MNRAS, 460, L30

Chatterjee S. et al., 2017, Nature, 541, 58

Connor L., Sievers J., Pen U.-L., 2016a, MNRAS, 458, L19

Connor L., Lin H.-H., Masui K., Oppermann N., Pen U.-L., Peterson J. B., Roman A., Sievers J., 2016b, MNRAS, 460, 1054

Cordes J. M., Lazio T. J. W., 2002, preprint (astro-ph/0207156)

Cordes J. M., Wasserman I., 2016, MNRAS, 457, 232

DeLaunay J. J. et al., 2016, ApJ, 832, L1

Dennison B., 2014, MNRAS, 443, L11

Falcke H., Rezzolla L., 2014, A\&A, 562, A137

Gehrels N., 1986, ApJ, 303, 336

Inoue S., 2004, MNRAS, 348, 999

Ioka K., 2003, ApJ, 598, L79

Karuppusamy R., Stappers B. W., van Straten W., 2010, A\&A, 515, A36

Kashiyama K., Ioka K., Mesaros P., 2013, ApJ, 776, L39

Keane E. F., Petroff E., 2015, MNRAS, 447, 2852

Keane E. F. et al., 2016, Nature, 530, 453

Lorimer D. R., Bailes M., McLaughlin M. A., Narkevic D. J., Crawford F., 2007, Science, 318, 777

Luan J., Goldreich P., 2014, ApJ, 785, L26

Lyubarsky Y., 2014, MNRAS, 442, L9

Lyutikov M., Burzawa L., Popov S. B., 2016, MNRAS, 462, 941

Masui K. et al., 2015, Nature, 528, 523

Newburgh L. B. et al., 2016, Proc. SPIE, 9906, 99065X

Ng C. et al., 2017, preprint (arXiv:1702.04728)

Petroff E. et al., 2015, MNRAS, 454, 457 
Petroff E. et al., 2016, PASA, 33, e045

Rajwade K. M., Lorimer D. R., 2017, MNRAS, 465, 2285

Ravi V., Shannon R. M., Jameson A., 2015, ApJ, 799, L5

Ravi V. et al., 2016, Science, 354, 1249

Scholz P. et al., 2016, ApJ, 833, 177

Shannon R. M., Ravi V., 2017, ApJ, 837, L22

Spitler L. G. et al., 2014, ApJ, 790, 101

Spitler L. G. et al., 2016, Nature, 531, 202

Tendulkar S. P. et al., 2017, ApJ, 834, L7

Thornton D. et al., 2013, Science, 341, 53

Totani T., 2013, PASJ, 65, L12

Vedantham H. K., Ravi V., Hallinan G., Shannon R., 2016, ApJ, 830, 75
Williams P. K. G., Berger E., 2016, ApJ, 821, L22

Wright E. L., 2006, PASP, 118, 1711

Xu J., Han J. L., 2015, Res. Astron. Astrophys., 15, 1629

Yao J. M., Manchester R. N., Wang N., 2017, ApJ, 835, 29

Zhou B., Li X., Wang T., Fan Y.-Z., Wei D.-M., 2014, Phys. Rev. D, 89, 107303

\section{APPENDIX A: POSITIONAL COORDINATES OF FRBS 160317,160410 AND 160608}

The coordinates of the FRB localization ellipses in Fig. 4 are given in Table A1.

Table A1. Sky coordinates of the three UTMOST FRBs. For each FRB, the first two columns are the J2000 right ascensions (RAs) and declinations (DECs) of the coordinates of the line defining the major axis of the $3 \sigma$ FRB localization contours in Fig. 4, given in units of degrees. The third column gives the probability of the event occurring at this point along the localization arc.

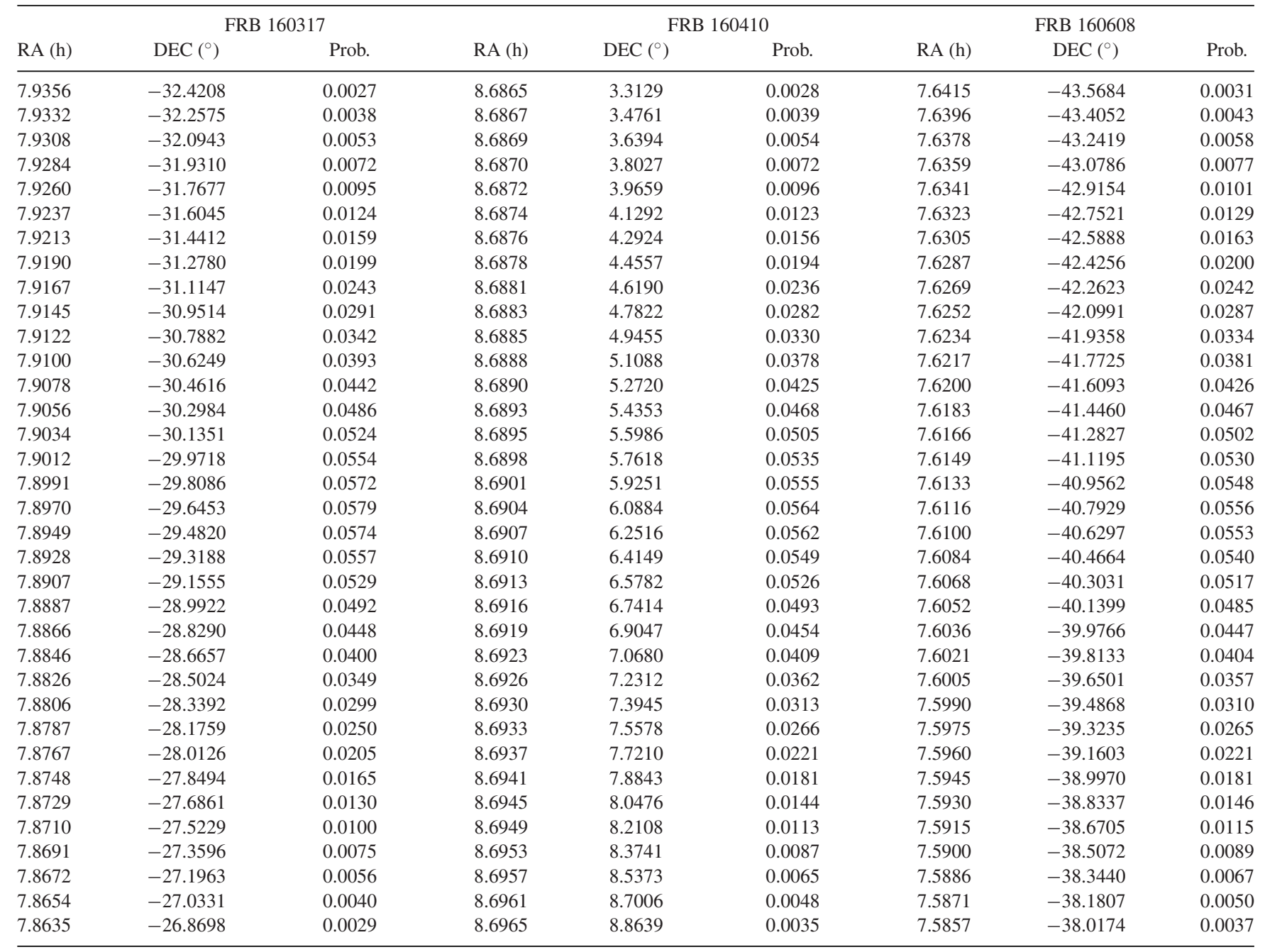

This paper has been typeset from a $\mathrm{T}_{\mathrm{E}} \mathrm{X} / \mathrm{L} \mathrm{A} \mathrm{E} \mathrm{X}$ file prepared by the author. 